\title{
On the Length Scale Dependence of Microscopic Strain by SANS
}

\section{S. Westermann, W. Pyckhout-Hintzen,* and D. Richter}

Forschungszentrum J ülich, Institut für Festkörperforschung, Postfach 1913, D-52425 J ülich, Germany

\section{E. Straube}

Martin-Luther-Universität HalleWittenberg, Fachbereich Physik, D-06099 Halle, Germany

\section{S. Egelhaaf and R. May}

Institute Laue Langevin, B.P. 156, F-38042 Grenoble Cedex 9, France

Received August 14, 2000

\begin{abstract}
We present a SANS study on the length scale dependence of chain deformation patterns in dense cross-linked elastomers. Three different polyisoprene networks of long primary block-copolymer chains of the HDH-type were analyzed. The total length of the primary chains is identical, while the length of the deuterated middle block was varied in order to cover several length scale regimes of interest. The scattering data are analyzed in the frame of the tube model of rubber elasticity in combination with the random phase approximation (RPA), which is used in order to account for the interchain correlations. We show experimentally that for the longest labeled path the rubber elastic response is dominated by both cross-link and entanglement contributions, whereas it is of clear phantomlike chain behavior if the labeled middle block of the chain is shorter than the distance between two successive entanglements, i.e., the tube diameter. We further propose schemes to quantify nonaffine deformations on various length scales and chain scissioning processes during the radical cross-linking process.
\end{abstract}

\section{Introduction}

The study of rubberelastic phenomena in polymer networks has greatly benefitted from the use of smallangle neutron scattering (SANS). Exploiting the large scattering contrast that can be achieved by blending protonated chains with the deuterated counterpart, a fundamental understanding of microscopic deformations and topol ogical constraints of label ed paths in a rubber is within reach. It was shown that the tube model of rubber elasticity is the most successful one for arriving at a consistent description of scattering and stressstrain data, ${ }^{1-6}$ and at the same time it is very well suited for application in finite el ement techniques. ${ }^{7} \mathrm{~A}$ detailed relation between the macroscopic deformation of a sample of rubber and the microscopic deformation of a network chain was established. The central aspect of this mean-field theory is that the free energy is governed not only by the effect of the chemical crosslinks but al so by the contribution of the entanglements due to the mutual uncrossability of the chains. ${ }^{5,8}$ Their influences on the fluctuations of the chain are modeled in terms of a deformation-dependent harmonic potential, the amplitude of which is the tube diameter ${ }^{9}$ that varies with the square root of the applied strain.

In a recent paper, studies on polyisoprene homopolymer systems randomly cross-linked by peroxides were extended to the case of a HDH isotopic triblock copolymer with a deuterated middle block connected to two equally sized protonated wings. ${ }^{10}$ There, the end-to-end distance of the labeled path was much longer than the average strand length between two cross-links. With respect to the homopolymer studies, this HDH system has the advantage that the label ed path may be firmly embedded into the macroscopic network, and no direct scattering contributions from labeled dangling chains ends, as is the case for fully labeled paths, will occur.

In this study, we present further results on $\mathrm{HDH}$ triblock copolymers of the same total chain length as before but containing shorter label ed middle blocks. This allows for a very sensitive microscopic determination of local deformations and the constraining potential at different length scales. The length scale under investigation is the size of the label ed block. The main control parameters determining the deformation and fluctuation behavior on this length scale are the average chain length between cross-links and the tube diameter. It is hoped in this way to contribute experimentally to the not completely understood question of the origin of nonaffine deformations in rubbers ${ }^{11}$ and to obtain from the dependence of the scattering behavior on the welldefined control parameters new information about chain deformation on this level.

As al ready pointed out ${ }^{10,12}$ the scattering of a network made from HDH triblock copolymers using DCP as cross-linker differs at small q considerably from the scattering of the melt before cross-linking. This was attributed to chain scission during cross-linking, and will be discussed in detail below. Other factors such as density fluctuations due to finite compressibility and also cluster formation during the first stages of crosslinking cannot account for the observed effects. The influence of chain scission reactions was considered in a first very rough approach considering one random scission per chain and calculating then the RPA corrections for the original triblocks and the split components independently. This corresponds in reality to a violation of the incompressibility condition of the system as a whole, but it was shown that this simple picture al ready all owed a successful description of the scattering results of triblock networks in terms of scission parameters. Here, the scission kinetics will be rigorously treated, assuming a completely random scissioning degradation. The scattering properties are obtained applying the standard RPA approach ${ }^{13}$ to this multicomponent chain system. This corresponds to the assumption that at early stages of cross-linking ( below 
Table 1. Summary of the Chemical Characteristics of the Discussed Networks Made of Polyisoprene HDH Triblock Copolymer Chains ${ }^{\mathrm{a}}$

\begin{tabular}{cccrcccc}
\hline label & $\mathrm{M}_{\mathrm{w}, \text { tot }}$ & $\phi_{\mathrm{D}}$ & \multicolumn{1}{c}{$\mathrm{M}_{\mathrm{c}}$} & $\mathrm{w}_{\mathrm{gel}}$ & instrument & $\mathrm{w}_{\mathrm{t}}$ & $\mathrm{M}_{\mathrm{n}} / \mathrm{M}_{0}$ \\
\hline T1 & 135000 & 0.36 & 6500 & 0.92 & PAXY + NG7 & 0.53 & 0.37 \\
T2 & 127000 & 0.08 & 21100 & 0.92 & PAXY + D22 & 0.70 & 0.24 \\
T3 & 140000 & 0.03 & 8000 & 0.913 & KWS1 + D22 & 0.95 & 0.30
\end{tabular}

a The first column defines a short label to simplify discussions in the text. $M_{w}$ and $M_{c}$ are in $\mathrm{g} / \mathrm{mol}$ and estimated error bars on $\mathrm{w}_{\mathrm{t}}$ and $\mathrm{M}_{\mathrm{n}} / \mathrm{M}_{0}$ are on the order of $5 \%$.

the gel point) a chain scission leads to a loss of correlation which can be observed whereas later (above the gel point) the chains are almost fixed in space and a further scission does not influence the scattering properties.

\section{Experimental Section}

Narrow molecular weight distributed polyisoprene is obtained by the anionic polymerization technique using sec-B uL $\mathrm{i}$ as the initiator in an apolar solvent, $n$-hexane. I sotopic triblock structures of the HDH-type were achieved from sequential addition of carefully weighed amounts of the respective monomers under the necessary precautions to limit chain degradation due to impurities and to preserve symmetry in the wings. Molecular weights of the single blocks were determined by GPC from samples taken out of the glass-sealed reactor by sealing off at predesignated constrictions. No asymmetry larger than $5 \%$ in absol ute molecular weights was observed, and the overall polydispersities were less than 1.05 . The labeled center fraction was varied between 3 and 36 vol $\%$ and confirmed by D-NMR. Sample characteristics are summarized in Table 1. The total molecular weights were further confirmed by low-angle laser-light-scattering experiments and membrane osmometry.

Randomly cross-linked networks were prepared from the pure triblocks using dicumyl peroxide (DCP) as the cross-linker. Samples and DCP were dissolved in THF and evaporated in a Teflon mold over about 1 week under high vacuum to ensure solvent-free polymer. The molds were then closed, and crosslinking was started under argon atmosphere at a temperature $\mathrm{T}=160{ }^{\circ} \mathrm{C}$ for $2-3 \mathrm{~h}$ to ensure complete decomposition of the DCP. Elastic chain lengths, $M_{c}$, were calculated from the swelling degree in cyclohexane, thereby assuming volume additivity and are listed in Table 1 . The cross-link density in the samples was varied to allow the investigation of both local strains and topology contributions in different relative proportions. Networks were strained in a calibrated straining rig and extension ratios determined from reference marks on the sample.

SANS experiments on melts and corresponding networks were recorded at PAXY (LLB, Saclay, France), NG-7 (NIST, Washington, DC), D22 (ILL, Grenoble, France) and KWS1 (FZ, J ülich, Germany) using wavelengths $\lambda$ of respectively $8,7,10$, and $7 \AA$ and $\Delta \lambda / \lambda \sim 0.1-0.15$. The corresponding q ranges were $0.007-0.09,0.01-0.13,0.006-0.15$, and $0.005-0.16 \AA^{-1}$, respectively. Resolution effects were calculated not to affect the data in the different experiments and were neglected. ${ }^{14}$ Scattering intensities were corrected for empty beam, cell, and detector efficiency and absolutely calibrated against water, silica, or lupolen depending on the instrument used. Crosschecks yiel ded a very good agreement in the absol ute level of intensities. Incoherent backgrounds measured from fully protonated samples were subtracted, weighed with their volume fraction present. Sample thicknesses between 0.5 and $1 \mathrm{~mm}$ avoid multiple scattering contributions. Data reduction was carried out pixelwise. The measured cross sections $\mathrm{d} \Sigma / \mathrm{d} \Omega$ are related to the system structure factors $\mathrm{S}(\overrightarrow{\mathrm{q}})$ according to

$$
\frac{d \Sigma(\vec{q})}{d \Omega}=\frac{\left(a_{H}-a_{D}\right)^{2}}{v_{0}} \phi_{D} N_{D} S(\vec{q})
$$

$v_{0}, a_{H}$, and $a_{D}$ are the monomeric volume and respective scattering lengths of protonated and deuterated monomers. $\phi_{D}$ and $N_{D}$ are fraction and segment numbers of the labeled part. Principal axis data were obtained from an extrapolation in a Zimm approximation around the respective direction following

$$
S\left(q_{\|}, q_{\perp}\right)^{-1}=S\left(q_{\|}, 0\right)^{-1}\left(1+q_{\perp}^{2} R_{g, a p p}^{2} / 3\right)
$$

for, e.g., parallel direction. F or small q, the separation of axes is correct. The range of application, i.e., $\mathrm{qR}_{\mathrm{g}} \leq 1$ thereby is respected. The method is superior over sector-binned data and physically correct, whereas the latter is not and depends strongly on the opening angle particularly in perpendicular direction. ${ }^{15}$ The proposed extrapolation to zero opening angle to relieve this problem on the other hand, is, however, questionable. For the two-dimensional data, fits in the whole 2d-detector plane are made. Similar to our previous publication, a splining of the theoretical function onto a grid of size $26 \times 26$ in one quadrant of the $\left(q_{\|}, q_{\perp}\right)$ plane is sufficient to accurately evaluate the true function values at any $\left(q_{\|}, q_{\perp}\right)$ pair.

\section{Theory and Concepts}

3.1. Scattering Properties of Triblock Networks. As in our previous work, ${ }^{10}$ the Warner-Edwards approach $^{16}$ is adopted as theoretical basis for the calculation of the structure factor of a labeled path in a network. For a single labeled path well connected into a permanent network with tube confinement without extra restrictions due to the cross-linking points, the chain form factor $S(\vec{q}, \lambda)$ is written as

$$
\begin{gathered}
\mathrm{S}(\overrightarrow{\mathrm{q}}, \lambda)=2 \int_{0}^{1} \mathrm{dx} \int_{0}^{\mathrm{x}} \mathrm{dx} \mathrm{X}_{\mu} \exp \left[-\left(\mathrm{Q}_{\mu} \lambda_{\mu}\right)^{2}\left(\mathrm{x}-\mathrm{x}^{\prime}\right)-\right. \\
\mathrm{Q}_{\mu}{ }^{2}\left(1-\lambda_{\mu}{ }^{2}\right)\left\{\frac{\mathrm{d}_{\phi}{ }^{2}}{2 \sqrt{6} \mathrm{R}_{\mathrm{g}}{ }^{2}}\left(1-\exp \left[-\frac{\left(\mathrm{x}-\mathrm{x}^{\prime}\right)}{\mathrm{d}_{\phi}^{2} / 2 \sqrt{6} \mathrm{R}_{\mathrm{g}}^{2}}\right]\right)\right\}
\end{gathered}
$$

where $R_{g}$ is the radius of gyration of the labeled path and $\mathrm{Q}_{\mu}=\mathrm{q}_{\mu} \mathrm{R}_{\mathrm{g}}$ is the component of the reduced scattering wave vector in the main axis system of the deformation tensor. $x$ and $x^{\prime}$ are dimensionless contour length coordinates extending over the labeled paths of the chains. $d_{\phi}=d_{0} \lambda_{\phi}{ }^{\nu}$ is the restricted root-mean-square displacement of the chain segments. The exponent $v=$ $1 / 2$ expresses the nonaffinity of the tube deformations with $\lambda_{\phi}^{2}=\lambda^{2} \cos ^{2} \phi+(1 / \lambda) \sin ^{2} \phi$ as an effective extension ratio ${ }^{17}$ into the observed direction $\phi . \phi$ is the angle between $\vec{q}$ and the parallel axis of strain. The first term corresponds to the scattering of the affinely deformed Gaussian chain, whereas the second accounts for the nonaffine contribution due to chain fluctuations.

The scale of affinity is an important aspect in the analysis of block copolymers. In the general case that the labeled part is shorter than the elastic chain length but larger than the entanglement mesh, deformations less than affine must be expected naturally. We will start with the simplest assumption that the average deformation on the length scale of the average distance between cross-links is affine. This assumption deviates from the idea 18 that the critical length of affinity is the entanglement length given by the tube diameter. Our 
assumption is mainly supported by the consideration that entanglements suppress transversal fluctuations only and therefore contour length fluctuations are not suppressed on this level. To treat subaffine deformations, the mean square fluctuations and the structure factors of a subchain of length $\mathrm{N}=\mathrm{N}_{\mathrm{c}}$ with $\mathrm{N}_{\mathrm{c}}$ being the mesh size are calculated described by the following conditional distribution function which corresponds to the above-discussed assumption

$$
p_{i j}\left(r_{i j} \mid \lambda\left\langle r_{O N}\right\rangle\right)=\frac{p_{0 i, 1}\left(r_{0 i}\right) p_{i j, 1}\left(r_{i j}\right) p_{j N, 1}\left(r_{j N}\right)}{p_{O N, 1}\left(r_{O N}\right)} p_{O N, \lambda}\left(r_{O N}\right)
$$

This distribution function corresponds to the case that the ends 0 and $\mathrm{N}$ are freely fluctuating with an extended mean square end-to-end distance of $\lambda^{2}\left\langle\mathrm{r}_{\mathrm{ON}}{ }^{2}\right\rangle$ as in the phantom model. The distribution functions

$$
p_{k l, \lambda}\left(r_{k l}\right)=\frac{1}{\left(2 \pi \lambda^{2}\left\langle r_{k 1}^{2}\right\rangle\right)^{3 / 2}} \exp \left(-\frac{3 r_{k l}^{2}}{2 \lambda^{2}\left\langle r_{k l}^{2}\right\rangle}\right)
$$

are equilibrium or distorted Gaussian distribution functions of the subchains or of the complete chain respectively with a mean square end-to-end distance of $\lambda^{2}\left\langle\mathrm{r}_{\mathrm{kl}}{ }^{2}\right\rangle$. The result

$$
\left\langle\mathrm{r}_{\mathrm{ij}, \lambda}{ }^{2}\right\rangle=\frac{\left[1-\left(|\mathrm{j}-\mathrm{i}| / \mathrm{N}_{\mathrm{c}}\right)\left(1-\lambda^{2}\right)\right]}{\lambda^{2}} \lambda^{2}\left\langle\mathrm{r}_{\mathrm{ij}, 1}{ }^{2}\right\rangle=\alpha \lambda^{2}\left\langle\mathrm{r}_{\mathrm{ij}, 1}{ }^{2}\right\rangle
$$

agrees with earlier results for phantom networks ${ }^{19}$ and an alternative approach to subaffine deformations by Rubinstein and Panyukov. ${ }^{11}$ The upper relation is consistent with the basic assumption that the shortest length scale to undergo affine deformational behavior is that connected to the length of the elastic chains located between two cross-links. For $\mathrm{j}-\mathrm{i}=\mathrm{N}_{\mathrm{c}}$, the factor between square brackets cancels out to 1 and the affine result is obtained.

The effective extension of a subchain is then simply controlled by its relative length

$$
\mathrm{f}_{\mathrm{c}}=|\mathrm{j}-\mathrm{i}| / \mathrm{N}_{\mathrm{c}}
$$

The contributions to the corresponding structure factor of a subchain are then obtained by replacing $\lambda^{2}$ in eq 3 by $\alpha \lambda^{2}$ as defined by eq 6 . Having introduced the corrections to the local strain in terms of the elastic strand length, we still have to distinguish between the case where the labeled path is well entangled and the phantom case. In latter situation, the structure factor is further modified by omitting the second term in eq 3.

For the investigated dense chain system of chemical identical chains the interchain correlations must be considered. In an RPA treatment for an incompressible melt, the partial structure factor for a 2-component system of $\mathrm{H}$ and $\mathrm{D}$ segments is written as ${ }^{13}$

$$
\mathrm{S}_{\mathrm{ij}, \mathrm{RPA}}=\mathrm{S}_{\mathrm{ij}, 0}-\frac{\sum_{\mathrm{h}=1}^{\mathrm{Nb}} \mathrm{S}_{\mathrm{ih}, 0} \times \sum_{\mathrm{I}=1}^{\mathrm{Nb}} \mathrm{S}_{\mathrm{lj}, 0}}{\sum_{\mathrm{i}, \mathrm{j}=1}^{\mathrm{Nb}} \mathrm{S}_{\mathrm{ij}, 0}}
$$

where $\mathrm{Nb}$ is the number of blocks in the copolymer.

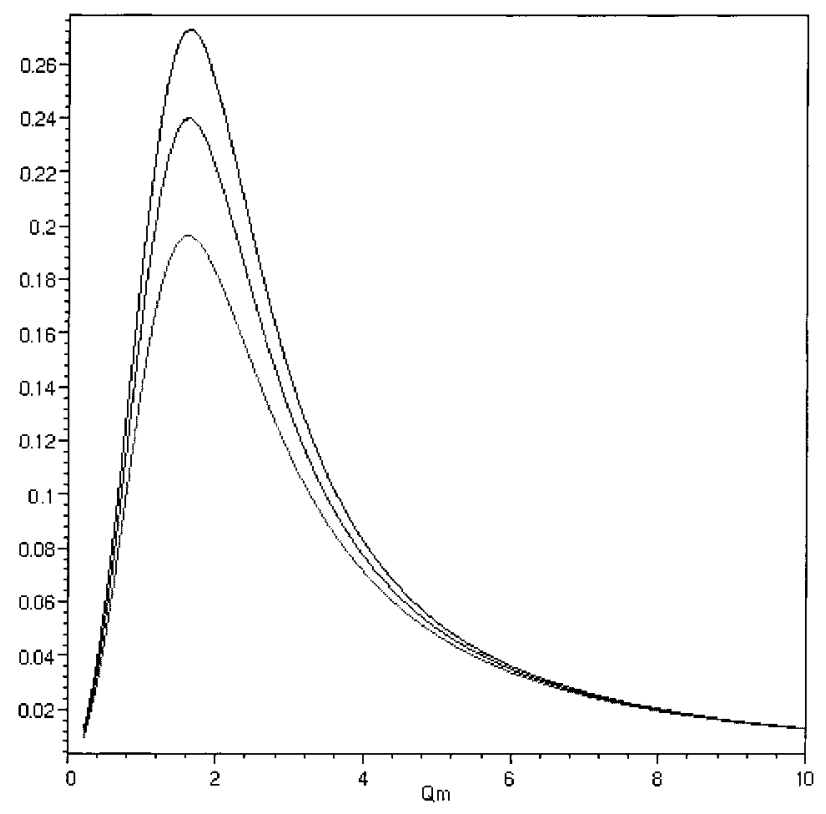

Figure 1. Key: lower, pure triblock; upper, symmetric fourarm star; middle, equimolar mixture of both. The x-axis is the rescaled scattering vector $\mathrm{qR}_{\mathrm{g}}$ for the case of $\mathrm{T} 1$.

$S_{i j, R P A}$ is the contribution from blocks $i$ and $j$ to the measured structure factor for interacting chains whereas the $\mathrm{S}_{\mathrm{ij}, 0}$ are the bare structure factors of the corresponding blocks without interaction. In triblock copolymers with deuterated middle blocks (conventionally numbered $i=j=2$ ) the sum in the denominator runs over the contributions $S_{12}, S_{22}$, and $S_{23}$ whereas in the nominator contributions from all blocks occur. Equation 8 can be recast simply in the better known Leibler notation. 20

The structure factor of the deformed network is determined based on the earlier proposed concept ${ }^{10}$ that the intrachain and interchain correlations in melts and undeformed networks are independent and their contributions to the system structure factor factorize. This is rigorous for dense melts. With the assumption that also for a deformed network the restricted fluctuations on the length scale of the tube dimensions are statistically independent of the long range correlations between the chains the structure factor for networks made of statistical ly cross-linked monodisperse HDH PI-triblock copolymers is written as

$$
\mathrm{S}_{\mathrm{tri}}(\overrightarrow{\mathrm{q}}, \lambda)=\mathrm{S}_{22, \mathrm{RPA}}(\overrightarrow{\mathrm{q}}, \lambda) / \mathrm{S}_{22,0}(\overrightarrow{\mathrm{q}}, \lambda) \times \mathrm{S}(\overrightarrow{\mathrm{q}}, \lambda)
$$

In this equation the ratio $S_{22, R P A}(\vec{q}, \lambda) / S_{22,0}(\vec{q}, \lambda)$ describes the q-dependent interchain correlations corrections between the middle blocks in the system and is responsible for the RPA peak structure. It is determined assuming affine deformation. Multiplication with the chain form factor $S(\vec{q}, \lambda)$ of the constrained chain of block length corresponding to $S_{22}$ then yields the measured structure factor of the system. The basic assumption of the RPA, i.e., the random flight statistics of the intrachain correlations in a dense melt, is the main support of this approach. It is rigorous for completely localized chains with $\mathrm{d}_{0}=0$ and a good approximation for $\mathrm{d}_{\phi}{ }^{2} / 2 \sqrt{6} \mathrm{R}_{\mathrm{g}}^{2} \ll 1$ where the effect of the constraints influences the scattering behavior at a $q$ range only where the interchain correlations are unimportant. This is also in agreement with the general assumption of the 
Warner-Edwards approach that the mean configurations of the chains in the undeformed network (or the configurations of the tube axis) can be described by a random walk distribution and fluctuations around these configurations are independent of the actual configuration. ${ }^{16}$ It should be noted that this approximation corresponds to the case that topological constraints dominate the fluctuations about average positions and that incompressibility effects may be neglected on this length scale.

For centrosymmetrical triblocks, the contributions to $S_{22, R P A}(\vec{q}, \lambda)$ in eq 8 read

$$
\begin{aligned}
\mathrm{S}_{11,0}= & \mathrm{S}_{33,0}=\frac{2 \mathrm{~N}_{\mathrm{w}}{ }^{2}}{\mathrm{Q}_{\mathrm{w}}{ }^{2}}\left[1-\left(1-\exp \left(-\mathrm{Q}_{\mathrm{w}}{ }^{2}\right)\right) / \mathrm{Q}_{\mathrm{w}}{ }^{2}\right] \\
\mathrm{S}_{12,0}= & \mathrm{S}_{23,0}= \\
& \frac{\mathrm{N}_{\mathrm{w}} \mathrm{N}_{\mathrm{m}}}{\mathrm{Q}_{\mathrm{w}}{ }^{2} \mathrm{Q}_{\mathrm{m}}{ }^{2}}\left[1-\exp \left(-\mathrm{Q}_{\mathrm{w}}{ }^{2}\right)\right]\left[1-\exp \left(-\mathrm{Q}_{\mathrm{m}}{ }^{2}\right)\right] \\
\mathrm{S}_{13,0} & =\frac{\mathrm{N}_{\mathrm{w}}{ }^{2}}{\mathrm{Q}_{\mathrm{w}}{ }^{4}}\left[1-\exp \left(-\mathrm{Q}_{\mathrm{w}}{ }^{2}\right)\right]^{2} \exp \left(-\mathrm{Q}_{\mathrm{m}}{ }^{2}\right) \\
\mathrm{S}_{22,0} & =\frac{2 \mathrm{~N}_{\mathrm{m}}{ }^{2}}{\mathrm{Q}_{\mathrm{m}}{ }^{2}}\left[1-\left(1-\exp \left(-\mathrm{Q}_{\mathrm{m}}{ }^{2}\right)\right) / \mathrm{Q}_{\mathrm{m}}{ }^{2}\right]
\end{aligned}
$$

$\mathrm{N}_{\mathrm{w}}$ and $\mathrm{N}_{\mathrm{m}}$ denote the numbers of statistical segments in a wing and in the middle part of the block copolymer

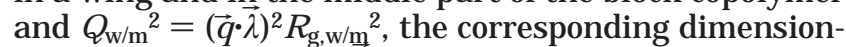
less scattering vectors. $\lambda$ is the (microscopic) deformation tensor.

3.2. Chain Scission. As will be shown experimentally below, the scattering of the un-cross-linked tribl ock melts under investigation is in excellent agreement with the predictions from eqs 8 and $10-13$, but as already outlined, ${ }^{10}$ networks made from bulk triblock systemsat least with DCP as cross-linker-show considerable nonzero scattering at small $\mathrm{q}$ which was related to random chain splitting during the cross-linking process. Assuming incompressibility it follows directly from the structure of eq 8 that, without allowing for changes of the lengths of the label ed paths, the property $S_{22, R P A}=$ 0 for $q=0$ remains unchanged at network formation independent of the details of the process. The reason for this behavior is the property $\mathrm{S}_{\mathrm{ij}, 0}(0)=\mathrm{N}_{\mathrm{i}} \mathrm{N}_{\mathrm{j}}$, which holds independent of the kind and length of connections between the blocks $i$ and $j$ of an isolated chain or of the same chain in a cluster or in a network. The formation of branched chains, of clusters and finally of the network leads only to small changes of position, shape, and height of the correlation peak. Model calculations for a mixture of triblock chains and stars formed from this triblock clearly show this behavior. The largest deviation occurs by cross-linking of the central segment of the triblock. Figure 1 presents the structure factors of the pure triblock system, of a system of symmetric 4-arm stars made from these triblocks and of equimolar mixture from both components. Nonzero intensity at q $=0$ in such a system can only arise if compressibility is allowed for ref 13. Especially we have to keep in mind, that additionally network inhomogeneities may be made responsible for scattering at small q if the system is not completely incompressible.

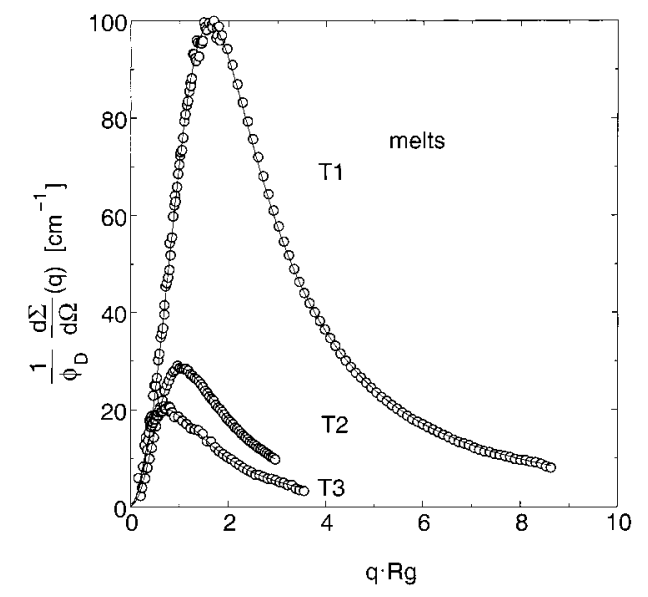

Figure 2. Radially averaged SANS data for un-cross-linked $\mathrm{T} 1, \mathrm{~T} 2$, and T3 samples in reduced and normalized representation. $R_{g}$ al ways denotes the radius of gyration of the label ed block.

Theoretical predictions by Brereton and Vilgis ${ }^{21}$ deal with this quenching effect for the extreme case of crosslinks which are fixed in space. Accordingly, these would be detectable at length scales at least 1 order of magnitude larger than the chain dimension which is outside of the investigated q range and very difficult to measure at all with SANS.

A MC simulation 22 of the system structure factor for a triblock melt and the randomly cross-linked system cross-linked with moderate cross-linking rate shows almost no difference for the small-q scattering and completely agrees with the RPA results for the incompressible triblock melt. By moderate cross-linking rate is meant that during the cross-linking process the average mean square displacements of chain segments are on the order of $R_{g}$ of the primary chains and therewith larger than the distance between cross-links. Consequently, if no significant density fluctuations can be observed for the investigated model system with a polymer volume fraction of $\sim 0.34$ and a slightly higher compressibility as the real system, the use of the RPA result for incompressible mel ts seems to be completely satisfied.

In this study, we will estimate the effect of chain scission within the RPA for the melt more quantitatively. As al ready pointed out in the Introduction the following considerations are necessary:

(1) The multicomponent structure of the system, which is the result of chain degradation at various stages of cross-linking, will change the scattering behavior at all q.

(2) Large scale inhomogeneities without changes of the chain structure will influence the scattering mainly at small q. ${ }^{12,23}$

Keeping this in mind, we will be able to distinguish between the building up and quenching of network fluctuations and the effect of chain scission. Consequently, as main criterion for the correctness of the chosen approach the equal agreement between theory and measured intensity over the whole q range will be decisive.

The choice of a suitable approximation for the generalization of eq 8 makes use of the result of model calculations in Figure 1 and of a comparison of measured intensities for un-cross-linked melts and networks from the same chains in Figures 2 and 3. Because there is no increase of intensity in comparison to the melt case 


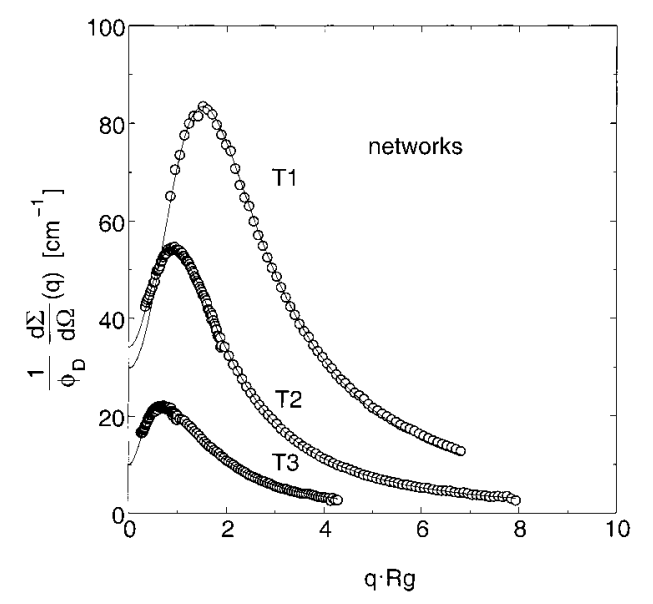

Figure 3. Fit to the SANS data of the network samples using the RPA structure factor modified for chain splitting. Same convention as in previous figure for $\mathrm{R}_{\mathrm{g}}$.

in the peak region it can be concluded that the formation of higher correlated branched structures is unimportant (it is obviously prevented by incompressibility). In the network case, a clear increase of intensity at smallest $q$ and also a slight increase at large $q$ can be detected. This is the typical result of an RPA description of a polydisperse ensemble obtained by scission. Taking this into account, we will approximate the intermolecular correlations by the RPA structure factor of an ensemble with an appropriate chosen degree of chain scission (i.e., the degree of chain scission at the gel point). The generalization of eq 8 for the degraded triblock at hand then reads

$$
\begin{gathered}
\mathrm{S}_{22, \mathrm{RPA}}=\left(\mathrm{N}_{\mathrm{t}} \mathrm{S}_{\mathrm{t}, 22}+\sum_{\mathrm{i}} \mathrm{N}_{\mathrm{d}, \mathrm{i}} \mathrm{S}_{\mathrm{d}, 22, \mathrm{i}}+\sum_{\mathrm{i}} \mathrm{N}_{\mathrm{mh}, \mathrm{i}} \mathrm{S}_{\mathrm{mh}, \mathrm{i}}\right)- \\
\left(\sum_{\mathrm{i}, \mathrm{j}} \sum_{\nu=1}^{3} \mathrm{~N}_{\mathrm{t}, \mathrm{ij}} \mathrm{S}_{\mathrm{t}, 2 v, \mathrm{ij}}+\sum_{\mathrm{i}, \mathrm{j}} \sum_{\nu=1}^{2} \mathrm{~N}_{\mathrm{d}, \mathrm{ij}} \mathrm{S}_{\mathrm{d}, 2 v, \mathrm{ij}}+\sum_{\mathrm{i}} \mathrm{N}_{\mathrm{mh}, \mathrm{i}} \mathrm{S}_{\mathrm{mh}, \mathrm{i}}\right) * \\
\left(\sum_{\mathrm{i}, \mathrm{j}} \sum_{\mu=1}^{3} \mathrm{~N}_{\mathrm{t}, \mathrm{ij}} \mathrm{S}_{\mathrm{t}, u 2, \mathrm{ij}}+\sum_{\mathrm{i}, \mathrm{j}} \sum_{\mu=1}^{2} \mathrm{~N}_{\mathrm{d}, \mathrm{ij}} \mathrm{S}_{\mathrm{d}, u 2, \mathrm{ij}}+\sum_{\mathrm{i}} \mathrm{N}_{\mathrm{mh}, \mathrm{i}} \mathrm{S}_{\mathrm{mh}, \mathrm{i}}\right) / \\
\left(\sum_{\mathrm{i}, \mathrm{j}} \sum_{\mu, v=1}^{3} \mathrm{~N}_{\mathrm{t}, \mathrm{ij}} \mathrm{S}_{\mathrm{t}, u v, \mathrm{ij}}+\sum_{\mathrm{i}, \mathrm{j}} \sum_{\mu \nu=1}^{2} \mathrm{~N}_{\mathrm{d}, \mathrm{ij}} \mathrm{S}_{\mathrm{d}, u v, \mathrm{ij}}+\right. \\
\left.\sum_{\mathrm{i}} \mathrm{N}_{\mathrm{mh}, \mathrm{i}} \mathrm{S}_{\mathrm{mh}, \mathrm{i}}+\sum_{\mathrm{i}} \mathrm{N}_{\mathrm{uh}, \mathrm{S}} \mathrm{S}_{\mathrm{uh}, \mathrm{i}}\right)
\end{gathered}
$$

The first term gives the contribution of the ideal polydisperse ensemble without interaction to the scattering. The structure factors in eq 14 are given by eqs 10-13 with appropriate segment numbers of the parts of the chains under consideration. The symbols in eq 14 denote the numbers $(\mathrm{N} .$.$) of chains with the ap-$ propriate structure factors (....) of one component, the subindexes denote the type of the chains (tri, di, marked (from the inner part) and unmarked homopolymers), $\mu$ and $v$ denote the part of the chain ( 2 is al ways used for the deuterated part, 1 and/or 3 for the wings), and $i$ and $j$ are the segment numbers in the wings of triblocks, in both parts of the diblocks and in the homopolymers, respectively.

Calculating the intermol ecular chain correlations by the RPA as described above implicitly means that the loss of intramolecular correlations by chain scission is taken into account only below the gel point. This assumption is justified by the consideration that scissioned chains remain correlated if scission after reach- ing the gel point occurs. ${ }^{23}$ It must be expected that the formed macroradicals due to the low mobility beyond the gel point will react with a group in the immediate vicinity and in this way conserve and fix the correlations (cage effect). This is supported al so by the observation, that the amount of sol fraction is distinctly lower than the values expected from the number-averaged molecular mass determined from scattering data.

Mainly to reduce the numerical effort for the description of scattering data the confinement parameter $\mathrm{d}_{\phi}{ }^{2} / 2 \sqrt{6} \mathrm{R}_{\mathrm{g}}{ }^{2}$ in the second exponential function in eq 3 is kept independent of chain length even for chains of different length after scission. Then the scattering factor for the multicomponent system will read

$$
\mathrm{S}_{\mathrm{tri}, \mathrm{sc}}(\overrightarrow{\mathrm{q}}, \lambda)=\mathrm{S}_{\mathrm{RPA}}(\overrightarrow{\mathrm{q}}, \lambda) / \mathrm{S}_{22,0}(\overrightarrow{\mathrm{q}}, \lambda) \times \mathrm{S}(\overrightarrow{\mathrm{q}}, \lambda)
$$

If the approximation for the confinement parameter would not be justified and factorization as in ref 15 is not possible, a considerably increased value for the tube diameter would show up to correct for the lack of description for the smallest chains due to the larger confinement parameter. The ratio $S(\vec{q}, \lambda) / S_{22,0}(\vec{q}, \lambda)$, which describes the fluctuations around mean configurations, is in this approximation independent of the polydispersity of the sample. The latter is completely contained in $\mathrm{S}_{\mathrm{RPA}}$. This approximation is slightly worse for the shortest chains, but the contribution of these chains to the scattering properties is only important for the intermolecular correlations (described by $\mathrm{S}_{\mathrm{RPA}}(\overrightarrow{\mathrm{q}}, \lambda)$ ) and therefore justified also for large degrees of chain splitting.

For the determination of the chain numbers $\mathrm{N}_{\text {... }}$ a set of kinetic equations was set up and solved numerically by iteration. The scission probabilities for each individual segment were taken identical and starting with a monodisperse distribution, the resulting distribution is determined by one single parameter. For convenience, this parameter is the fraction of remaining triblock chains $w_{t}$ (including polydisperse wings). The kinetic equations, balancing gain of chains, and loss of initial chains in the number of segments are given in the Appendix.

\section{Results}

4.1. The Reference HDH Copolymer Melts. Prior to discussing the network properties, the melt characteristics of the T1, T2, and T3 polymers (see Table 1) are summarized. Radially averaged SANS data (NG-7, PAXY, KWS1) are shown in Figure 2. To facilitate comparison, the coherent scattering intensities are normalized to their labeled volume fraction and are pl otted against the reduced scattering vectors $q R_{g}$. The scattering data in Figure 2 are well fitted to the RPA structure factor given by eq 15 . The radii of gyration of the labeled block were determined to $\mathrm{R}_{\mathrm{g}, \mathrm{T} \text { lmelt }}=(73.5$ $\pm 0.5) \AA, R_{g, T 2 m e l t}=(35.6 \pm 0.5) \AA$ and $R_{g, T 3 m e l t}=(22.1$ $\pm 0.5) \AA$ in good agreement to the expected values of 72,33 , and $21 \AA$ of the free chains assuming a characteristic ratio of $\mathrm{C}_{\infty}=5.1$ and $5 \%$ of 3,4 units for this particular microstructure. ${ }^{24}$ The lack of any detectable intensity at $q \rightarrow 0$ as well as the peak shape to the left of the maximum ascertains the monodispersity of the un-cross-linked neat materials. The deviation in $\mathrm{R}_{\mathrm{g}}$ may be ascribed to some possible polydispersity of the labeled part or in the exact volume fraction. 
4.2. I sotropic HDH Copolymer Networks. Figure 3 shows the experimental data for the undeformed networks together with the best fit according to eqs 3-15 in the same representation as the melts. The networks showed radii of gyration of the labeled blocks to be $R_{g, T 1}=(77.3 \pm 1.5) \AA, R_{g, T 2}=(38.7 \pm 1.2) \AA$, and $\mathrm{R}_{\mathrm{g}, \mathrm{T} 3}=(21.6 \pm 0.6) \AA$. In view of the comparison of data from different spectrometers, the correspondence between unperturbed chain dimensions in both melt and network state is satisfying and supports the validity of the above-discussed approximations. The agreement between experimental data and theory is almost of the same quality in the complete q range as in the melt case. Besides a similar peak structure the scattering of the networks is distinguished by finite scattering at zero $\mathrm{q}$.

The new parameter of the fitting procedure is $\mathrm{w}_{\mathrm{t}}$, representing the fraction of triblock chains with scissions in the wing part only during the cross-linking process. This choice is more appropriate than the description we proposed earlier. Additionally, the ratio $\mathrm{M}_{\mathrm{n}} / \mathrm{M}_{0}$ of number-averaged chain mass of the distribution to mass of the primary chains is used as characteristics of the scission process and al so given in Table 1. The very different numerical values for the fraction $w_{t}$ are due to the different lengths of the label ed parts. The ratio $\mathrm{M}_{n} / \mathrm{M}_{0}$ on the other hand serves as a measure of the scissions of the primary chains and yields comparable values as they should be for a randomly occurring process.

Recalling the discussions above, it should be noticed that the obtained characteristics of the scission process are determined after complete cross-linking but correspond to an intermediate stage of scission and crosslinking and are frozen in at least at the gel point. The different effectivity of the scission process on the loss of intrachain correlations and devel opment of large scale fluctuations below and above the gel point is the aim of a more elaborate analysis by the in situ examination of cross-linking and scissioning in a SANS + rheology experiment in a forthcoming study, ${ }^{23}$ and only the outcome is anticipated here. There it turns out clearly that the observed influence of chain splitting on scattering data is strongly reduced beyond the gel point at unchanged thermal conditions and therefore chemical kinetics. Therefore, the assumptions discussed above can be considered to be strongly supported, and evidence of the scission of chains here is obtained which cannot be determined otherwise by pure chemical methods 25 and will also be almost undetectable by spectroscopic methods, e.g., NMR. ${ }^{26}$

Resuming the results for undeformed networks, it can be concluded that the agreement between experimental values and theoretical curves is very satisfactory over the whole $q$ range and comparable intensities in the limit $\mathrm{q}=0$ are obtained. Therefore, the scattering behavior of the investigated networks follows the predictions of the used RPA approach. This conclusion is further supported by the fact that the reduction of the scattering intensity in the peak region very precisely corresponds to the prefactors foll lowing from our kinetic model and the used scattering theory.

4.3. HDH Copolymer Networks under Deformation. We restate that the networks which are studied here are now characterized in addition to the confinement parameter $d_{0}^{2} / R_{g}{ }^{2}$, by two more parameters: $M_{c}$ and $M_{D}$. The deuterated fraction $M_{D}$ or the corresponding length scale $R_{D}$ determines the length scale under

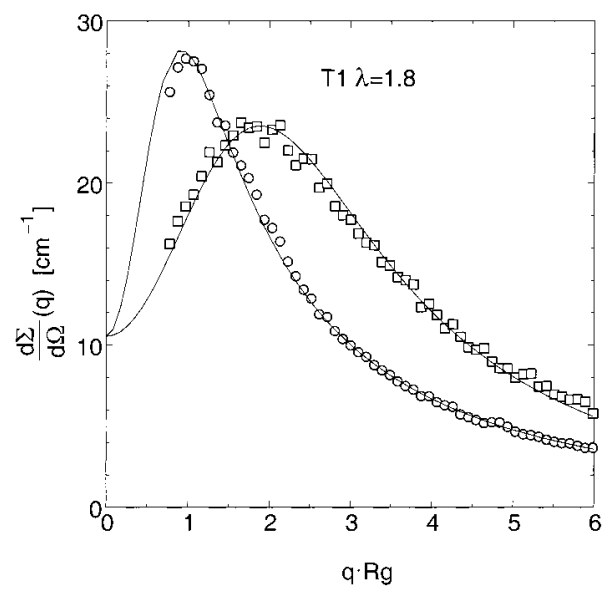

Figure 4. Axis data of the network sample of $\mathrm{T} 1$ at $\lambda=1.8$ using the RPA structure factor corrected for chain scission.

investigation and shows up in the confinement term via $R_{g}$, whereas the elastic chain mass, given by $M_{c}$ or $R_{c}$, determines the critical level at which the strain will be become affine. The tube diameter did not change as a function of $\mathrm{M}_{\mathrm{c}}$ in our previous investigations. Three cases are to be differentiated.

4.3.1. The Case $\mathbf{d}_{\mathbf{0}}<\mathbf{R}_{\mathbf{c}}<\mathbf{R}_{\mathbf{D}}$. From all triblock copolymer networks in this work, the T1 sample should behave similar to a typical homopolymer networks made from long primary chains, which are well cross-linked with the exception for the existence of relaxed chain end effects. This is the case of strong constraints. The tube model applies without exception and this was already proved. The end-to-end distance of the labeled path in T1 with $R_{g}=77 \AA$ is $R_{D} \approx 180 \AA$ and is longer than the tube diameter $d_{0}=42 \AA$, which may be considered as the corresponding spatial distance between two entanglements. $R_{D}$ is also larger than the average distance between two cross-links $M_{c}=6450 \mathrm{~g} / \mathrm{mol}, R_{c}=63 \AA$. Figure 4 presents both main directions at a deformation ratio $\lambda=1.8$. Structural parameters from the isotropic network were fixed. The intensity drop is perfectly predicted by the fitted distribution function. The size of the tube fitted is consistent with our former estimate, $42 \pm 1 \AA$, and no anomalies in the $q$ dependence observed.

4.3.2. The Case $\mathbf{d}_{\mathbf{0}}<\mathbf{R}_{\mathbf{D}}<\mathbf{R}_{\mathbf{c}}$. The network sample of $\mathrm{T} 2$ is expected to behave somewhat differently on strain for several reasons. The end-to-end distance $R_{D}$ $\approx 95 \AA$ (with $\mathrm{R}_{\mathrm{g}}=38.7 \AA$ ) is still larger than the tube dimension of $\mathrm{d}_{0}=42 \AA$ but now is less than the strand length between two chemical cross-links $\mathrm{R}_{\mathrm{c}} \approx 114 \AA$. From the relationship between $R_{D}$ and $R_{e}$, this particular case has the advantage that for a constrained chain the deformation of parts of chains within two cross-links can be investigated. The network was strained to ratios $\lambda_{\|}=\{1.35,1.6,2.0,2.5\}$. Figure 5 shows a 2 d-contour plot at the highest available strain and includes the best fit using the same tube diameter assuming an affine chain deformation on the microscopic level. A pronounced reduced experimental anisotropy in the parallel axis is observed and so the microscopic deformation is overestimated considerably.

The affine premise fails obviously, and the modification for weakly cross-linked label ed paths as introduced earlier is appropriate. A relaxed strain of the labeled part as discussed above must be considered. According to eqs 6 and 7 the observable deformation of the deuterated middle block is now the microscopic defor- 

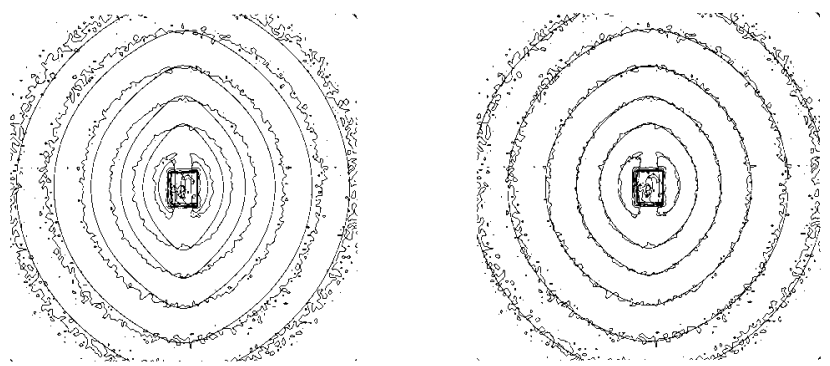

Figure 5. Two-dimensional contour plot of the SANS data measured from the T2 sample at highest strain $\lambda=2.5$ and comparison to the structure factor assuming an affinely deformed labeled path (left) and reduced strain (right). The data are in favor clearly with a subaffine deformation of the labeled path.

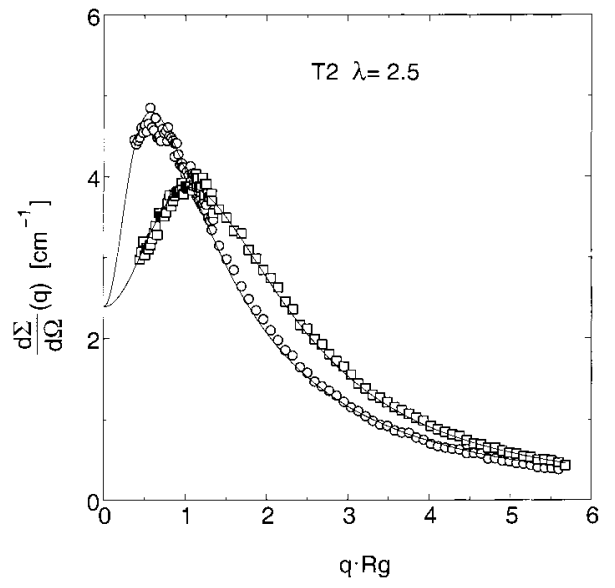

Figure 6. Corresponding axis data of Figure 5 for highest $\lambda$ $=2.5$ for sample T2.

mation $\lambda_{\mathrm{i}}{ }^{2}$ and a function of the ratio $\mathrm{f}_{\mathrm{c}}$ of the length of the labeled path to the length of a network chain between two cross-links, $f_{c}=M_{D} / M_{c}$. As the end-to-end distance of the deuterated middle block is still larger than the tube diameter the fluctuations of the labeled path will be still constrained due to the presence of the neighboring chains. Ther efore, the constraining potential and its deformation dependence need not to be modified for this strain reduction.

In the analysis of the scattering data $\mathrm{f}_{\mathrm{c}}$ is introduced as an additional fit parameter. The tube diameter $\mathrm{d}_{0}=$ $42 \AA$ was again kept fixed. The result of the 2-dimensional data fit now is shown in Figure 5 with $\mathrm{f}_{\mathrm{c}}=0.45$. On the basis of this modification, all strains were studied also by fits al ong the principal deformation axes. The corresponding axis fits for $\lambda=2.5$ are presented in Figure 6 . The results of all available strains indicate an average value of $\mathrm{f}_{\mathrm{c}}=0.41 \pm 0.01$ compared to $\mathrm{f}_{\mathrm{c}, \mathrm{th}}=$ $M_{D} / M_{C}=0.48$. This may be assigned to the uncertainty in $M_{D}$ (or $f$ ) and especially $M_{C}$, the latter being obtained from swelling measurements, but may also be caused by network defects which could enlarge the fluctuations and consequently reduce the local deformations. The case, in which one cross-link is situated within the label ed path and the other therefore not, is not considered in the estimation according to eqs 6 and 7. All this approximations would lead to an overestimation of $f_{C}$ for given values of $M_{D}$ and $M_{C}$.

It has to be added that the interchain structure factor $\mathrm{S}_{\mathrm{RPA}}(\overrightarrow{\mathrm{q}}, \lambda) / \mathrm{S}_{22,0}(\overrightarrow{\mathrm{q}}, \lambda)$ is calculated with the macroscopic extension ratio $\lambda$. This follows from the meaning of $\mathrm{S}_{\mathrm{RPA}}(\overrightarrow{\mathrm{q}}, \lambda) / \mathrm{S}_{22,0}(\overrightarrow{\mathrm{q}}, \lambda)$ describing the correlations between the centers of gravity of the chains which must be subjected to the macroscopic deformations.

4.3.3. The Case $\mathbf{d}_{\mathbf{0}} \approx \mathbf{R}_{\mathbf{D}}<\mathbf{R}_{\mathbf{c}}$. Finally, the interesting T3 sample is discussed. Here, the length of the labeled part $\left(R_{g}=21 \AA, R_{D} \approx 51 \AA\right.$ ) is comparable to the tube diameter $d_{0}=42 \AA$ and shorter than the average distance between two cross-links $\left(\mathrm{M}_{\mathrm{C}}=8000\right.$ $\left.\mathrm{g} / \mathrm{mol}, R_{c}=70 \AA\right)$. Again, following our observations for T2 the modifications for strain reduction of the labeled path must be used. However, the main difference with T2 and T1 is that for sample T3 strongly reduced constraints will be felt by the labeled segments. This becomes clear from considering Figure 7. It is impossible to obtain a reasonable description of the data with tubemodel scattering functions and tube parameters in the expected order of magnitude, especially in the perpendicular strain direction which is known to be the most sensitive to tube constrained fluctuation scattering. A satisfactory agreement was achieved using a phantom model. The structure factor which applies to the T3 sample is the structure factor of a phantom chain deformed by the microscopic deformation ratio $\lambda_{\mathrm{i}} .27,28$ The deformation of the labeled path is therefore a measure of the subaffinity inside a mesh of entanglements. The only relevant parameter is again $\mathrm{f}_{\mathrm{c}}$, defined above.

The T3 sample was investigated at three different extension ratios of $\lambda_{\|}=\{1.3,1.65,1.9\}$. Figure 8 shows a final $2 \mathrm{~d}$-fit as well as a principal axis fit for the highest deformation. A close match with the expected value of $f_{c, t h}=0.55$ is found in the $2 \mathrm{~d}$-data analysis, while the main axes systematically slightly overestimate the parameter by $15 \%$. It should be recalled here that the coherent scattering is roughly only about $5 \%$ above the incoherent level, and background subtraction on the basis of the labeled content of ( $3 \pm 2) \%$ (from NMR) therefore is very error prone. In the extrapolation to the axes to gain reasonable statistics, systematic deviations may have been introduced by the assumption of the $\mathrm{q}^{2}$ dependence which may be not fulfilled in these cases especially at small q due to the finite parasitic scattering. Also, for statistical reasons no two-dimensional data analysis was performed for the small q region, because also here contour plots are insensitive in view of the extremely flat curvature of the scattering profile.

Nevertheless, the data clearly prove that the deuterated middle block is still well-strained microscopically and behaves like a typical phantom chain. Every attempt to describe the data using a constraining potential with the typical value of $d_{0}$ failed.

Since the cross-link density of the T3 sample is comparable to that of the T1 sample, the studies on the T1 copolymer networks witness the crossover from the constrained chain behavior to a phantomlike chain behavior. This is in strong anal ogy with the well-known Rouse-to-reptation crossover transition ${ }^{9,29}$ in the description of polymer dynamics and may give additional insights of the typical length scales governing this process.

\section{Conclusion}

Experiments that were conducted on networks consisting of protonated and deuterated chain mixtures in the past, were repeated with isotopic block copolymers of the HDH type. The SANS patterns turned out to be very sensitive to scission processes upon cross-linking 

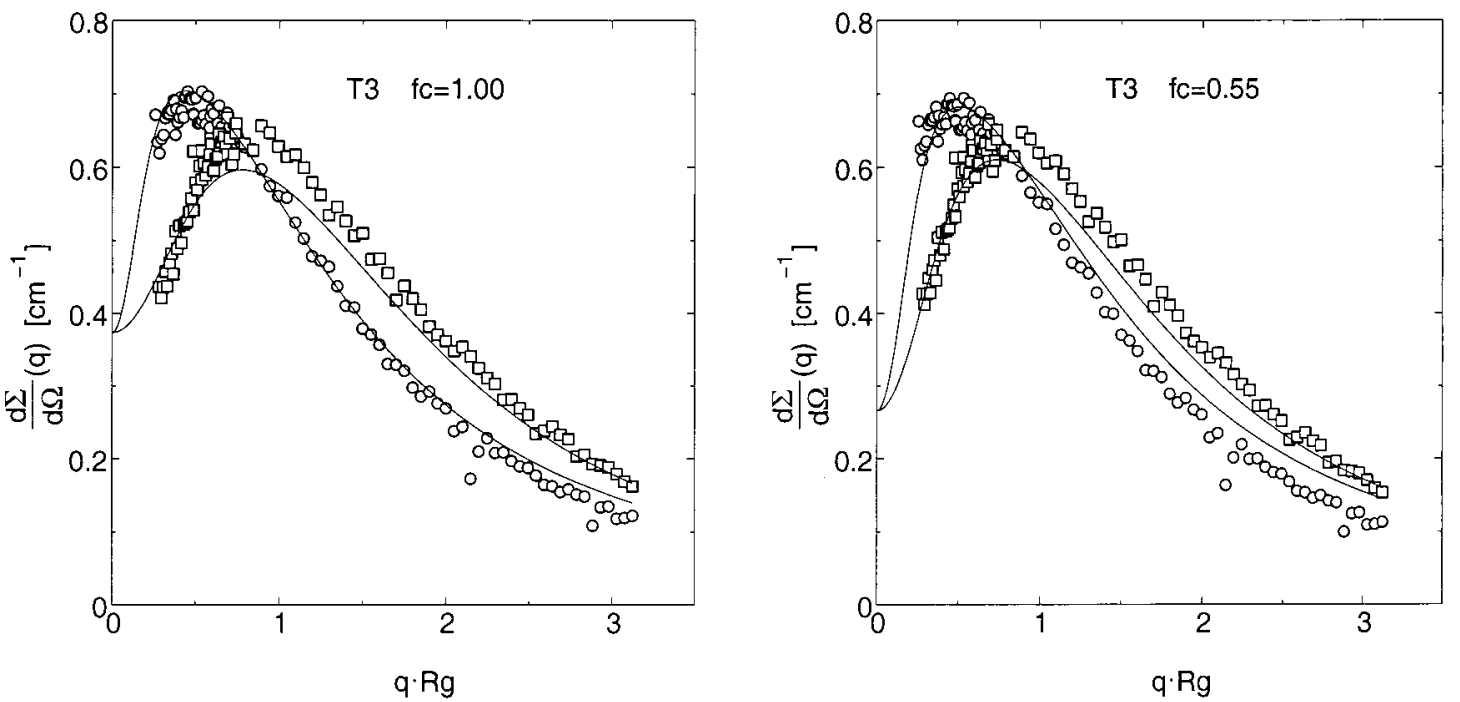

Figure 7. Comparison of the SANS data obtained from the T3 sample at a deformation of $\lambda=1.9$ with the theoretical structure factor of a labeled constrained path. Left: an affine displacement of the primitive path is assumed, which fails as well as the correction for the reduced strain shown on the right.
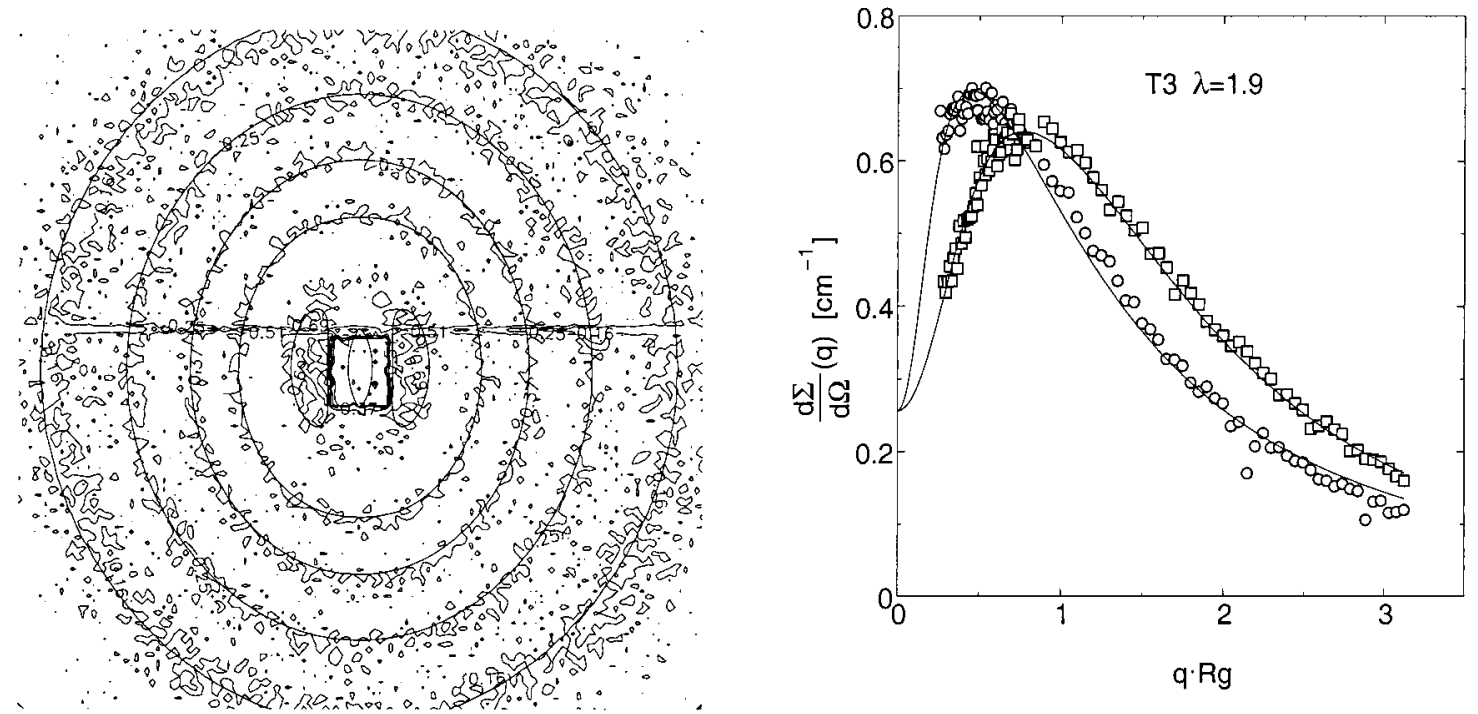

Figure 8. 2D-axis and main axis fits of the SANS data obtained from the T3 sample at $\lambda=1.9$ and comparison to the scattering function of a phantom chain. A good agreement over the entire q-interval $0.5 \leq \mathrm{qR}_{\mathrm{g}} \leq 3$ is found.

with the common dicumylperoxide, DCP. An RPA approach that takes into account all correlations within chains and chain scission products was proposed in this work and perfectly describes the isotropic networks. The structure factor within the tube model of Straube and Heinrich is derived and allows the experimental verification of microscopic deformation and the network topology under strain. Through variation of the length of the labeled parts of the triblocks the length scale of deformation is controlled. The transition from a phantomlike behavior to the constrained-chain behavior which is observed for the first time in a static experiment is compared to the famous Rouse-to-reptation transition. The scale of affinity was found to be set at the elastic strand length whereas a continuous reduction in affinity of strain is proposed for lengths smaller than the latter.

Finally, it should be remarked that our results concern the physics of networks made by vulcanization from long primary chains. These networks show al ways lozengic scattering patterns in the deformed state. The success of the tube model proves that fluctuations
Table 2

\begin{tabular}{ll}
\hline label & \multicolumn{1}{c}{ definition } \\
\hline$N_{t}$ & no. of unscissioned triblocks \\
$N_{t, i j}$ & no. of triblocks with polydisperse wings \\
$N_{d, i j}$ & no. of diblocks with polydisperse h- and d-blocks \\
$N_{m h, i}$ & no. of labeled homopolymers \\
$N_{u h, i}$ & no. of unlabeled homopolymers \\
$\mathrm{p}_{\mathrm{i}}$ & distribution function of the unlabeled part in di- \\
& $\quad$ and triblocks with $\sum_{\mathrm{i}} \mathrm{p}_{\mathrm{i}}=1$
\end{tabular}

beyond the scale of the tube dimensions are absent. It may therefore be concluded that the "butterfly" patterns observed in numerous experiments are due to the development of fluctuations during deformation caused by network defects which do not exist in networks from long primary chains.

\section{Appendix: Scission Kinetics in HDH Copolymers}

For the description of the scission process, see Table 2 for the variables.

The kinetic equations for the scission of a $\mathrm{N}_{0}$ triblock chains of total chain length $n$ and the volume fraction 
of the labeled symmetric middle block $f$ then read as follows.

Triblocks. The number of triblock dhains with lengths $\mathrm{i}$ and $\mathrm{j}$ in the wing parts is given by

$$
\mathrm{N}_{t, \mathrm{ij}}=\mathrm{N}_{\mathrm{t}} \mathrm{p}_{\mathrm{i}} \mathrm{p}_{\mathrm{j}}
$$

where the two distribution functions $p_{i}$ and $p_{j}$ account for statistically independent scission events in the wing parts.

The kinetic equation for the polydisperse triblocks reads

$$
\begin{aligned}
& \frac{d N_{t, i j}}{d t}=w_{s}\left[-(f n+i) N_{t, i j}+\sum_{k=i+1}^{n(1-f) / 2} N_{t, k j}-j N_{t, i j}+\right. \\
& \left.\sum_{l=j+1}^{n(1-f) / 2} N_{t, i l}\right]
\end{aligned}
$$

The factorization according to eq 16 leads to

$$
\frac{d N_{t}}{d t}=-w_{s} f n N_{t}
$$

and

$$
\frac{d p_{i}}{d t}=w_{s}\left[-i p_{i}+\sum_{k=i+1}^{n(1-f) / 2} p_{k}\right]
$$

Diblocks. For the polydisperse diblock copolymers

$$
\mathrm{N}_{\mathrm{d}, \mathrm{ij}}=\mathrm{N}_{\mathrm{d}, \mathrm{i}} \mathrm{p}_{\mathrm{j}}
$$

holds, with $p_{j}$ as above and the kinetic equation for $\mathrm{N}_{\mathrm{d}, \mathrm{i}}$ to

$$
\frac{d N_{d, i}}{d t}=w_{s}\left[-i N_{d, i}+\sum_{k=n_{i}+1}^{n f} N_{d, k}+2 N_{t}\right]
$$

Labeled Homopolymers. For the label ed homopolymers the equation reads

$$
\frac{d N_{m h, i}}{d t}=w_{s}\left[-i N_{m h, i}+2 \sum_{k=i+1}^{n f} N_{m h, k}+\sum_{k=i+1}^{n f} N_{d, k}\right]
$$

Unlabeled Homopolymers. Finally the equation for the unlabeled homopolymers is given by

$$
\frac{d N_{u h, i}}{d t}=w_{s}\left[-n_{i} N_{u h, i}+2 \sum_{k=i+1}^{n(1-f) / 2} N_{u h, k}+2 N_{m} \sum_{k=i+1}^{n(1-f) / 2} p_{k}\right]
$$

Starting Conditions. At the beginning the system is characterized by the following set of variables:

$$
\begin{gathered}
\mathrm{N}_{\mathrm{t}}=\mathrm{N}_{0} \\
\mathrm{~N}_{\mathrm{d}, \mathrm{i}}=0 \\
\mathrm{~N}_{\mathrm{mh}, \mathrm{i}}=0 \\
\mathrm{~N}_{\mathrm{uh}, \mathrm{i}}=0 \\
\mathrm{p}_{\mathrm{i}}=1, \quad \text { for } \mathrm{n}_{\mathrm{i}}=\mathrm{n}_{\mathrm{n}}(1-\mathrm{f}) / 2 \\
\mathrm{p}_{\mathrm{i}}=0, \text { otherwise }
\end{gathered}
$$

\section{References and Notes}

(1) Deam, R. T.; Edwards, S. F. Philos. Trans. R. Soc. London, Ser. A 1976, 280, 543.

(2) de Gennes, P. G. Scaling Concepts in Polymer Physics; Cornell University Press: I thaca, NY, 1979.

(3) Doi, M.; Edwards, S. F. The Theory of Polymer Dynamics; Clarendon: Oxford, England, 1986.

(4) Edwards, S. F.; Vilgis, T. A. Rep. Prog. Phys. 1988, 51, 243 297.

(5) Heinrich, G.; Straube, E.; Helmis, G. Adv. Polym. Sci. 1988, $85,33-87$.

(6) Marzocca, A.; Cerveny, S.; Raimondo, R. J . Appl. Polym. Sci. 1997, 66, 1085.

(7) Heinrich, G.; Kaliske, M. Comput. Theor. Polym. Sci. 1997, 7, 227.

(8) Everaers, R.; Kremer, K. Macromolecules 1995, 28, 7291.

(9) Richter, D.; Butera, R.; Fetters, L. J .; Huang, J. S.; Farago, B.; Ewen, B. Macromol ecules 1992, 25, 6156.

(10) Westermann, S.; Urban, V.; Pyckhout-Hintzen, W.; Richter, D.; Straube, E. Macromolecules 1996, 19, 6165.

(11) Rubinstein, M.; Panyukov, S. Macromolecules 1997, 30, 8036.

(12) Westermann, S.; Pyckhout-Hintzen, W.; Thyagarajan, P.; Wosniak, D.; Richter, D.; Straube, E. World Scientific Publ.: Singapore, 1999.

(13) Kim, J. K.; Kimishima, K.; Hashimoto, T. Macromolecules 1993, 26, 125-136.

(14) Pedersen, J.J . Phys. IV 1993, 3, 491.

(15) Boue, F. Adv. Polym. Sci. 1987, 82, 47.

(16) Warner, M.; Edwards, S. F. J . Phys. A. 1978, 11, 1649.

(17) Mildner, D. F. R. Macromolecules 1983, 16, 1760.

(18) Mueller, R.; Picot, C.; Zang, Y.; Froehlich, D. Macromolecules $1990,23,2577$

(19) Kloczkowski, A.; Mark, J .; Erman, B. Comput. Polym. Sci. $1992,2,8$.

(20) Leibler, L.; Benoit, H. Polymer 1981, 22, 195

(21) Brereton, M.; Vilgis, T.J .Phys. 1 Fr. 1992, 2, 581.

(22) Straube, E.; Pyckhout-Hintzen, W.; Richter, D. To be published, 2000

(23) Botti, A.; Pyckhout-Hintzen, W.; Richter, D.; Straube, E. Submitted for publication, 2000.

(24) Hadjichristidis, N.; Xu, Z.; Fetters, L.; Roovers, J. J . Polym. Sci., Polym. Phys. Ed. 1982, 20, 743.

(25) Capla, M.; Borsig, E. Eur. Pol. J . 1980, 16, 611.

(26) Mori, M.; Koenig, J . Rubber Chem. Technol. 1995, 68, 551.

(27) J ames, H.; Guth, E. J . Chem. Phys. 1943, 11, 455.

(28) J ames, H.J . Chem. Phys. 1947, 15, 651.

(29) Kremer, K.; Crest, G.; Carmesin, I. Phys. Rev. Lett. 1988, 61, 566.

MA0014259 